\title{
If you stock it, will they buy it? Healthy food availability and customer purchasing behaviour within corner stores in Hartford, CT, USA
}

\author{
Katie S Martin ${ }^{1, *}$, Erin Havens ${ }^{2}$, Katie E Boyle ${ }^{2}$, Gregory Matthews ${ }^{3}$, \\ Elizabeth A Schilling ${ }^{2}$, Ofer Harel ${ }^{3}$ and Ann M Ferris ${ }^{2}$ \\ 'Department of Allied Health Sciences, University of Connecticut, 263 Farmington Avenue, MC 6229, \\ Farmington, CT 06030-6229, USA: ${ }^{2}$ Center for Public Health \& Health Policy, University of Connecticut, \\ East Hartford, CT, USA: ${ }^{3}$ Department of Statistics, University of Connecticut, Storrs, CT, USA
}

Submitted 3 March 2011: Accepted 17 November 2011: First published online 10 January 2012

\begin{abstract}
Objective: Literature on food environments has expanded rapidly, yet most research focuses on stores and community characteristics without integrating customer-level data. The present study combines customer shopping behaviour with store food inventory data.

Design: Face-to-face interviews were conducted with customers shopping in corner stores to measure food shopping behaviour, household food security and demographics. Store inventories were conducted to measure availability of healthy food in corner stores. Multilevel logistic regression models estimated the probability of customers purchasing a food item given the availability of that item in the store.

Setting: Nineteen corner stores in Hartford, CT, USA, average size $669 \mathrm{ft}^{2}\left(62 \cdot 15 \mathrm{~m}^{2}\right)$. Subjects: Sample of 372 customers.

Results: The majority of customers were Black or Hispanic (54\% and $40 \%$, respectively) and $61 \%$ experienced food insecurity. For each additional type of fruits or vegetables available in the store, the estimated odds of a customer purchasing fruits increased by $12 \%(P=0.03)$ and the odds for purchasing vegetables increased by $15 \%(P=0 \cdot 01)$. Customers receiving the Supplemental Nutrition Assistance Program (SNAP) were 1.7 times as likely to purchase fruit as those not receiving SNAP $(P=0 \cdot 04)$. Greater availability of reduced-fat milk was not associated with increased likelihood of customers purchasing reduced-fat milk.

Conclusions: There is a positive association between fruit and vegetable variety and the probability that a customer purchases fruits and vegetables. Increasing the selection of produce in corner stores may increase their consumption by foodinsecure and low-income residents at risk for health disparities. These findings have implications for future store interventions and food policies.
\end{abstract}

Keywords
Corner stores
Customers
Healthy food
Diet is a modifiable risk factor for leading health concerns in the USA such as diabetes, CVD, cancer and obesity ${ }^{(1)}$. Health disparities exist for these chronic conditions, particularly among minority and low-income families ${ }^{(2,3)}$. Food insecurity, defined as limited or uncertain availability of nutritionally adequate foods ${ }^{(4)}$, has been linked with adult obesity, particularly in women ${ }^{(5-7)}$. Possible explanations include the fact that high-fat/high-energy food costs less than healthy food ${ }^{(8)}$, and that healthy, affordable food is less available in low-income urban neighbourhoods ${ }^{(9,10)}$. Recent attention has focused on the importance of local food environments that can contribute to or help prevent health disparities.
Literature on food environments has expanded rapidly, documenting that a lack of healthy, affordable food exists in low-income, minority neighbourhoods compared with wealthier, non-minority suburbs ${ }^{(9-13)}$. The wide availability of energy-dense snack foods in corner stores may contribute to obesity ${ }^{(14)}$. Associations exist between the presence of different types of food stores and dietary patterns and diet quality ${ }^{(15-18)}$. According to the national Healthy Corner Store Network, a 'corner store' is defined as a small-scale store that sells a limited selection of foods and other products ${ }^{(19)}$, and we use this definition in the current study.

The majority of research on corner stores has focused on store-level and ignored customer-level data. Limited 
research has examined purchasing habits among customers within corner stores. Borradaile et al. conducted an observational study of elementary students and measured their snack purchasing behaviour in corner stores ${ }^{(20)}$. Gittelsohn et al. evaluated a corner store intervention and its impact on customer purchases ${ }^{(21)}$. Few studies have examined the demographic characteristics of customers who shop in corner stores, measured their shopping behaviour, or linked store food availability to customer purchasing habits within the store. The present study begins to fill this gap.

The research reported herein contributes to the literature on food environments by combining customer shopping behaviour with store food inventory data. The goal of the study is to examine whether healthy food availability is associated with customer purchasing behaviour. We also examined the demographic characteristics of customers shopping in corner stores, including household food security and participation in food assistance programmes, and their typical shopping patterns.

\section{Methodology}

The city of Hartford, CT is an important focus for food environment research due to high rates of poverty and diet-related diseases. The poverty rate in Hartford in 2009 was $32 \%$, and $39 \%$ among children ${ }^{(22)}$. The population is predominantly Hispanic (41\%) and Black (37\%). Based on a random sample of Hartford residents, 31\% reported being diagnosed with hypertension, 13\% reported a diagnosis of diabetes and $35 \%$ of respondents were obese ${ }^{(23)}$.

\section{Customers}

The current study was conducted on a convenience sample of 372 customers shopping in nineteen corner stores in Hartford, CT. Inclusion criteria included being a resident of Hartford, over 18 years of age, and customers were asked if they did the majority of food shopping for their family. Researchers approached customers as they entered the store to ask if they were willing to participate in a research study related to food shopping, and if so, face-to-face interviews were conducted in the stores. Interviews were conducted at different times of the day and different days of the week to measure typical food shopping behaviour, household food security and household demographics. We did not collect information on customers who refused to participate, but the main reason for non-participation was lack of time.

Food shopping behaviour questions included where customers shop for food and how often, whether they participate in the Supplemental Nutrition Assistance Program (SNAP, formerly food stamps) and the Special Supplemental Nutrition Program for Women, Infants, and Children (WIC), and if so, if they use their SNAP or WIC benefits in the store. Customers were asked if they bought items at the store from a list of food products. The question stem was: 'Thinking about the foods that you bought at this store for you and the people you live with during the past three months, did you buy the following foods?' The food items included: (i) fresh fruits (six types and 'other' category); (ii) canned fruit; (iii) fresh vegetables (five types and 'other' category); (iv) canned and frozen vegetables; (v) types of milk (whole, $2 \%, 1 \%$ or skimmed); and (vi) types of bread (whole-wheat or white). The food items were informed by qualitative data from five focus groups with corner store customers in Hartford conducted in 2007 (K Martin, unpublished results).

Household food security was measured using the US Department of Agriculture (USDA) Food Security Module, which consists of eighteen questions that ask about a household's experiences with food insufficiency during the previous 12 -month period ${ }^{(24)}$. Responses were coded into the following categories using the USDA standardized labels: (i) high, (ii) marginal, (iii) low and (iv) very low food security. Responses were then dichotomized into food secure (levels i and ii) and food insecure (levels iii and iv) using USDA standard labels.

Customer demographic information included age, gender, education, employment, ethnicity, car ownership, marital status, and whether a member of the household has diabetes or high blood pressure. Customer data collection took place from March to May 2009.

\section{Corner stores}

We obtained a list of all grocery stores in the city of Hartford from Dun \& Bradstreet, a commercial marketing firm (http://www.dnb.com). We merged this with data on Hartford stores certified to accept coupons for the WIC programme (data obtained monthly from Connecticut Department of Public Health). The current study was part of a multi-phase, longitudinal study to evaluate a communitybased initiative targeting corner stores in Hartford, CT. The sampling frame included 123 stores with average sales of \$US 207000 and an average of 2.5 employees.

For the present study, corner stores are defined as small, independent food stores less than $2500 \mathrm{ft}^{2}\left(232 \mathrm{~m}^{2}\right)$ based on field measurements. By contrast, medium-sized grocery stores (such as Sav-A-Lot or C-Town) are approximately $15000 \mathrm{ft}^{2}\left(1395 \mathrm{~m}^{2}\right)$ and generally stock a limited number of custom-branded high-volume food items at a discount ${ }^{(25)}$. Large supermarkets (such as Stop \& Shop or Kroger) range from 40000 to $80000 \mathrm{ft}^{2}$ (3716 to $7432 \mathrm{~m}^{2}$ ) and in addition to groceries typically include delicatessens, bakeries, pharmacies, general merchandise and often banks. The nineteen stores in the present sample are defined as small corner stores. Gas stations, liquor stores and drug stores that sell food were excluded. Customers were asked how frequently they shop at small corner stores, medium-sized grocery stores and large supermarkets. 


\section{Sample size}

Data for the present study were part of a larger longitudinal study developed to examine the impact of a community-based programme over time. Power calculations were originally made to determine the sample sizes needed to detect differences in intervention conditions related to that programme. Data for the present paper are from baseline measurements. Because the study used a cluster correlated design in which observations were nested within customers which were nested within stores, power calculations were determined using Optimal Design software version $1.55^{(26)}$, a program developed to assist with power calculations for multilevel studies. We determined that a target sample of at least 350 customers within at least fourteen stores was needed to compare differences between stores. We oversampled to accommodate attrition.

\section{Store inventories}

Store inventories were conducted to measure availability and quality of healthy food in corner stores, using a modified version of the Nutrition Environment Measures Survey in Stores (NEMS-S) ${ }^{(27)}$. The revised instrument included availability and quality of fresh fruits and vegetables, canned and frozen fruits and vegetables (a prescribed list of fruits and vegetables plus space to write in additional types for both fresh and canned), whole grains and reduced-fat dairy products. The proportion of all milk by container count that is reduced-fat ( $2 \%$ fat or less) was calculated from the inventory data.

Pairs of researchers conducted the store inventories to measure availability of foods within the store, and also square footage using a laser measurer (Stanley FatMax Tru Laser). Two sets of square foot measurements from the pair of researchers were averaged together to calculate store size. The inventory had high inter-rater reliability (Cronbach's $\alpha=0 \cdot 84-0 \cdot 99$ ). Store inventories took place from January to February 2009. The University of Connecticut Institutional Review Board approved the protocol for the study. Both customers and store owners signed informed consents, and were paid \$US 5 for study participation.

\section{Data analyses}

Data were analysed using the SPSS statistical software package version 18 (SPSS Inc., Chicago, IL, USA) and the HLM (Hierarchical Linear Modeling) software program version 7 (Scientific Software International Inc., Lincolnwood, IL, USA). Descriptive statistics were calculated for customer demographics and store characteristics. Bivariate analyses were run to examine associations between customer demographics, store characteristics and purchasing behaviour using $\chi^{2}$ tests for dichotomous variables and Spearman correlations for continuous variables. Variables at the customer level included car ownership, ethnicity (dummy variables were created for Hispanic and
Black), high-school education, employment, gender, age (as continuous variable), food security and whether the household receives SNAP or WIC. Variables at the store level included store size (continuous square feet) and WIC certification.

To estimate the probability of customers purchasing a food item given the availability of that item in the store, multilevel logistic regression models using a Bernoulli distribution with a random intercept were estimated. The estimation procedure used was full maximum likelihood with Adaptive Quadrature. We modelled the probability of customers purchasing: (i) fresh fruit related to the variety of fruit available in the store; (ii) fresh vegetables related to the variety of vegetables available in the store; and (iii) reduced-fat milk given the proportion of reduced-fat milk in the store. Each model controlled for car ownership, receiving SNAP, employment, ethnicity, high-school education and age. The models were estimated as follows:

Level-1 model

$$
\begin{gathered}
\text { Prob }\left(\text { BuyFruit }_{i j}=1 \mid \beta_{j}\right)=\phi_{i j} \\
\log \left[\phi_{i j} /\left(1-\phi_{i j}\right)\right]=\eta_{i j} \\
\eta_{i j}=\beta_{j}+\gamma_{10} \times\left(\text { OwnCar }_{i j}\right)+\gamma_{20} \times\left(\text { SNAP }_{i j}\right) \\
+\gamma_{30} \times\left(\text { Working }_{i j}\right)+\gamma_{40} \times\left(\text { Hispanic }_{i j}\right)+\gamma_{50} \\
\times\left(\text { Black }_{i j}\right)+\gamma_{60} \times\left(\text { HighSchool }_{i j}\right)+\gamma_{70} \times\left(\text { Age }_{i j}\right)
\end{gathered}
$$

Level-2 model

$$
\beta_{0 j}=\gamma_{00}+\gamma_{01} \times\left(\text { FruitVariety }_{j}\right)+u_{0 j}
$$

where BuyFruit $_{i j}$ is 1 if the $i$ th person in the $j$ th store purchases fruit and 0 if they do not; $\gamma_{10}$ to $\gamma_{70}$ are the fixed effects for the customer-level characteristics; and $\beta_{0 j}$ is the random effect to account for the second level in our model - the store-level data. $\gamma_{00}$ and $\gamma_{01}$ are the fixed intercept and fixed effect for FruitVariety Fnd $_{j}$ and $u_{0 j}$ is the variability across stores.

\section{Results}

\section{Customer characteristics}

The average age of customers was $37 \cdot 7$ years, and $84 \%$ of the sample was female, see Table 1 . The majority of customers were Black or Hispanic (54\% and $40 \%$, respectively). Over a third (35\%) of customers had less than a highschool degree. Over half (57\%) were currently unemployed, compared with a city-wide average of $16 \%{ }^{(28)}$. Only one in five customers (20\%) owned a car, compared with a city-wide average of $41 \%$. Over two-thirds (70\%) of the sample were currently receiving SNAP. Among households with a child under 5 years of age, over half (56\%) currently received WIC.

Sixty-one per cent of customers experienced food insecurity, including $26 \%$ with low food security and $35 \%$ 
Table 1 Household demographics of sampled customers shopping in corner stores, Hartford, CT, USA

\begin{tabular}{lrr}
\hline Characteristic & $n$ & $\%$ \\
\hline Total sample & 372 & 100 \\
Household demographics & 312 & $84 \cdot 1$ \\
$\quad$ Female & 294 & $79 \cdot 9$ \\
$\quad$ Not married & 228 & $61 \cdot 3$ \\
Have children & 119 & $32 \cdot 0$ \\
Have children under 5 years of age & & \\
Ethnicity & 199 & $53 \cdot 5$ \\
Black & 149 & $40 \cdot 1$ \\
Hispanic & 24 & $6 \cdot 4$ \\
Other & & \\
Education & 130 & $35 \cdot 0$ \\
Less than high-school degree & 157 & $42 \cdot 4$ \\
High-school degree/GED & 84 & $22 \cdot 6$ \\
Some college or higher & 215 & $56 \cdot 6$ \\
No adult employed & 73 & $20 \cdot 0$ \\
Own a car & 258 & $69 \cdot 5$ \\
Receive SNAP/food stamps & 66 & $55 \cdot 5$ \\
Receive WIC (with children under 5 years of age) & & \\
Food security & 77 & $21 \cdot 6$ \\
$\quad$ Food secure & 63 & $17 \cdot 6$ \\
Marginal food security & 93 & $26 \cdot 1$ \\
Low food security & 124 & $34 \cdot 7$ \\
Very low food security & 78 & $21 \cdot 0$ \\
Self-reported diabetes in household & 119 & $32 \cdot 2$ \\
Self-reported high blood pressure in household & & \\
\hline
\end{tabular}

GED, General Educational Development; SNAP, Supplemental Nutrition Assistance Program; WIC, Special Supplemental Nutrition Program for Women, Infants, and Children.

with very low food security, such that adults often skipped meals and cut back on the size and quality of their children's meals. In addition, $21 \%$ of customers selfreported that they or a member of their household had diabetes and $32 \%$ had high blood pressure.

\section{Store characteristics}

Among the nineteen corner stores in the present sample, the average size was $669 \mathrm{ft}^{2}\left(62 \cdot 15 \mathrm{~m}^{2}\right.$; median $572 \mathrm{ft}^{2}$ $\left.\left(53 \cdot 14 \mathrm{~m}^{2}\right)\right)$. Thirty-seven per cent of stores were certified to accept coupons for WIC. On average, stores carried $4 \cdot 2$ (SD $2 \cdot 4$ ) types of fresh fruits and $6 \cdot 1(\mathrm{SD} 4 \cdot 1)$ types of fresh vegetables, and $74 \%$ of stores had reduced-fat milk available. Average length of store ownership was 7 years.

\section{Customer shopping bebaviour}

The majority of customers in the sample (52\%) said they bought most of their food at medium-sized grocery stores, see Table 2. Sixty-one per cent of the sample said they shop at large supermarkets once a month or less, while $32 \%$ shop at corner stores nearly every day. Shopping habits were correlated with customer demographics. Employment, education and car ownership were all significantly associated with more frequent trips to large supermarkets $(P<0 \cdot 01)$. Increased age, receiving SNAP and food insecurity were negatively associated with shopping at large supermarkets $(P=0 \cdot 05,<0 \cdot 01$ and $<0 \cdot 01$, respectively).
Table 2 Food purchasing behaviour of sampled customers shopping in corner stores, Hartford, CT, USA

\begin{tabular}{lrr}
\hline & $n$ & $\%$ \\
\hline $\begin{array}{l}\text { Location buy most of their food } \\
\text { Large supermarket (over } 40000 \mathrm{ft}^{2} \text { ) }\end{array}$ & 148 & $40 \cdot 8$ \\
Medium grocery (approx. $15000 \mathrm{ft}^{2}$ ) & 188 & $51 \cdot 8$ \\
$\quad$ Small corner store (less than $2500 \mathrm{ft}^{2}$ ) & 27 & $7 \cdot 4$ \\
How often shop at corner store & 123 & $34 \cdot 1$ \\
$\quad$ Once or twice per month & 121 & $33 \cdot 5$ \\
Once or twice per week & 117 & $32 \cdot 4$ \\
$\quad$ Every day & & \\
Typical purchases at corner store & & \\
Bread & 323 & $87 \cdot 8$ \\
$\quad$ Bread of any kind & 170 & $52 \cdot 6$ \\
$\quad$ Whole-wheat bread & & \\
Milk & 319 & $86 \cdot 7$ \\
$\quad$ Milk of any kind & 180 & $48 \cdot 9$ \\
$\quad$ Reduced-fat milk (2\% or less fat) & 306 & $83 \cdot 2$ \\
Snacks & & \\
Fruit & 230 & $62 \cdot 5$ \\
$\quad$ Fresh fruit & 172 & $46 \cdot 6$ \\
$\quad$ Canned fruit & 105 & $28 \cdot 5$ \\
$\quad$ Frozen fruit & & \\
Vegetables & 187 & $50 \cdot 7$ \\
$\quad$ Fresh vegetables & 197 & $53 \cdot 4$ \\
$\quad$ Canned vegetables & 118 & $32 \cdot 0$ \\
$\quad$ Frozen vegetables & & \\
\hline
\end{tabular}

Customers said they typically buy milk (87\%), snacks (such as chips, candy or ice cream) (83\%), fruit (70\%) and bread $(70 \%)$ at their corner store. Among these, approximately half bought healthier versions such as whole-wheat bread (53\%) and reduced-fat milk (49\%). Among those participating in food assistance programmes, $90 \%$ of SNAP recipients said they use their benefits at their corner store and $54 \%$ of WIC recipients said they use their benefits at WIC-certified corner stores.

\section{Purchasing behaviour with store characteristics}

When controlling for potential covariates (listed in Methodology section), greater fruit variety was associated with an increased likelihood that customers purchase fruit at the store (see Table 3 for results from the multilevel regression models). For a one unit increase in the availability of varieties of fresh fruit, the likelihood that customers purchase fruit increased by $12 \%(P=0 \cdot 03)$. Those receiving SNAP were significantly more likely to purchase fruit compared with those not receiving SNAP $(\mathrm{OR}=1 \cdot 72$; $95 \%$ CI $1 \cdot 02,2 \cdot 91 ; P=0 \cdot 04)$.

Vegetable availability was also associated with an increased likelihood that customers purchase vegetables in the store. For a one unit increase in the number of vegetables available in the store, the estimated odds of a customer purchasing vegetables increased by $15 \%$ $(P=0 \cdot 01)$. Increased age was significantly associated with purchasing fresh vegetables $(P<0 \cdot 01)$ in the corner store.

Having a greater proportion of milk that was reducedfat did not increase the likelihood that customers purchased reduced-fat milk in the store (results not shown). 
Table 3 Results of multilevel logistic regression models predicting purchases among sampled customers shopping in corner stores, Hartford, CT, USA

\begin{tabular}{lccc}
\hline & OR & $95 \% \mathrm{Cl}$ & $P$ value \\
\hline Purchase fruit & & & \\
Intercept & 1.04 & $0.32,3.48$ & 0.94 \\
Fruit variety in store & 1.12 & $1.01,1.25$ & 0.03 \\
Own a car & 0.97 & $0.55,1 \cdot 73$ & 0.93 \\
Receive SNAP & 1.72 & $1.02,2 \cdot 91$ & 0.04 \\
Employed & 0.84 & $0.50,1.39$ & 0.49 \\
High-school education & 0.60 & $0.36,1.01$ & 0.06 \\
Hispanic & 1.01 & $0.39,2.59$ & 0.98 \\
Black & 0.92 & $0.37,2.32$ & 0.87 \\
Age & 1.00 & $0.98,1.02$ & 0.83 \\
Purchase vegetables & & & \\
Intercept & 0.59 & $0.17,2 \cdot 12$ & 0.39 \\
Vegetable variety in store & 1.15 & $1.07,1.23$ & 0.01 \\
Own a car & 1.13 & $0.61,2.08$ & 0.70 \\
Receive SNAP & 1.10 & $0.63,1.93$ & 0.73 \\
Employed & 1.07 & $0.63,1.85$ & 0.79 \\
High-school education & 0.63 & $0.37,1.09$ & 0.10 \\
Hispanic & 0.99 & $0.37,2.72$ & 0.99 \\
Black & 0.64 & $0.24,1.74$ & 0.39 \\
Age & 1.05 & $1.02,1.07$ & 0.01 \\
\hline
\end{tabular}

SNAP, Supplemental Nutrition Assistance Program.

\section{Discussion}

Lack of supermarkets paired with high poverty rates and health disparities highlight the need to explore food availability and food purchasing habits among urban residents. The majority of customers in the present sample said they bought most of their food at medium-sized grocery stores which tend to have custom brands and lower prices than large supermarkets, yet they shop frequently at small corner stores to buy staple foods and snacks. Customers in our sample have high rates of food insecurity and diet-related health conditions including diabetes and high blood pressure. Those who shop most frequently at corner stores have higher rates of food insecurity and lower socio-economic status (no car, limited education and employment) than those shopping more frequently at large supermarkets, exacerbating their risk for health disparities. This highlights the importance of corner store interventions and local food policies to reach those most in need.

In order to address health disparities, a healthy food environment requires both supply and demand from customers and store owners. Most of the literature on corner stores focuses on the supply side. The present study adds to the literature by combining customer purchasing information with food availability in the stores where they shop. Our findings show that customers are more likely to purchase fruits and vegetables when there is a wider variety in the store. Corner store owners may be concerned about lack of demand from customers and limited shelf space and refrigeration to stock fresh produce ${ }^{(29)}$. The present results indicate that demand is related to supply, which may encourage store owners to stock a larger supply of produce.
While our study has several strengths, there are some notable limitations. The study was conducted in a nonrepresentative sample of stores in one medium-sized city and therefore results are limited to the study area. It was conducted among a convenience sample of customers, and it is possible that customers who declined to participate have different purchasing habits compared with study participants. Shopping behaviour is based on self-report rather than direct observations or sales data; therefore some caution in interpretation is advised. Food prices were also not collected for the study but they very likely contribute to purchasing decisions. Data are also cross-sectional and causality cannot be implied.

\section{Implications}

Acknowledging the need for further confirmation of the direction and cause of effect in the present cross-sectional study, increasing the number and selection of produce in corner stores may increase their purchase and ultimately consumption by food-insecure and low-income residents who need them most. The majority of customers in the present sample who participate in SNAP and WIC utilize their benefits in corner stores, and those who receive SNAP have increased odds of purchasing fresh fruit in the store. The additional purchasing power and nutrition education associated with SNAP may encourage low-income families to purchase fruit compared with similar families not receiving SNAP. With the recent changes to the WIC food package including fruits, vegetables, whole grains and low-fat milk (which occurred after the present data were collected), additional research is needed to document changes to availability of these foods within stores and customer purchasing habits among WIC clients.

There appear to be regional differences in corner store sizes. For example, several studies mention a definition of corner stores as less than $200 \mathrm{ft}^{2}\left(18.58 \mathrm{~m}^{2}\right)^{(14,20)}$. No stores in the present sample were less than $200 \mathrm{ft}^{2}$, stressing the importance of defining and measuring store size. Square foot measurements are feasible to administer and future research should take different store sizes into consideration.

Classifying areas with few large supermarkets as food 'deserts' may overlook the availability of healthy foods that exist within corner stores, the purchasing habits among low-income customers and the purchasing power of SNAP that contributes to healthy food shopping within corner stores. Many households that are most at risk for diet-related diseases have less access to large supermarkets ${ }^{(16,17)}$. Increasing the availability and affordability of healthy foods within corner stores is needed to serve those most in need.

The present study fills a gap in the literature by combining store-level food availability with customer purchasing habits within those stores. Corner store customers in the current sample have high rates of chronic diseases 
and food insecurity. Consumption of healthy foods can help decrease the risk for these diseases. Our results show that when stores carry a greater variety of fruits and vegetables, customers tend to buy them. These results may have practical benefits for organizations and municipalities working with corner stores in other urban environments.

\section{Acknowledgements}

Funding for the present study was generously provided by a grant from the Patrick and Catherine Weldon Donaghue Medical Research Foundation. There is no conflict of interest to declare. K.S.M. and A.M.F. were Co-Principal Investigators of the study. K.S.M. was involved with study design, data collection, analyses and manuscript writing. E.H. and K.E.B. assisted with data collection and analyses. G.M. assisted with the multilevel regression analyses and O.H. assisted with data analysis. E.A.S. conducted sample size estimates. All authors contributed to writing and editing the manuscript.

\section{References}

1. McKenna M, Taylor W, Marks J et al. (1998) Current issues and challenges in chronic disease control. In Chronic Disease Epidemiology and Control, pp. 1-26. Washington, DC: American Public Health Association.

2. Centers for Disease Control and Prevention (2009) Differences in Prevalence of Obesity Among Black, White, and Hispanic Adults - United States, 2006-2008. http://www. cdc.gov/obesity/data/trends.html\#Race (accessed April 2010).

3. Ogden C, Carroll M, Curtin L et al. (2006) Prevalence of overweight and obesity in the United States, 1999-2004. JAMA 295, 1549-1555.

4. Life Sciences Research Office (SA Anderson, editor) (1990) Core indicators of nutritional status for difficult-to-sample populations. J Nutr 120, Suppl. 11, 1559S-1600S.

5 Holben D (2006) Position of the American Dietetic Association: food insecurity and hunger in the United States. J Am Diet Assoc 106, 446-458.

6 Wilde P \& Peterman J (2006) Individual weight change is associated with household food security status. J Nutr 136, 1395-1400.

7. Martin K \& Ferris A (2007) Food insecurity and gender are risk factors for obesity. J Nutr Educ Behav 39, 31-36.

8. Drewnowski A (2004) Obesity and the food environment: dietary energy density and diet costs. Am J Prev Med 27, 3 Suppl., 154-162.

9. Jetter KM \& Cassady D (2006) The availability and cost of healthier food alternatives. Am J Prev Med 30, 38-44.

10. Horowitz C, Colson K, Hebert P et al. (2004) Barriers to buying healthy foods for people with diabetes: evidence of environmental disparities. Am J Public Health 24, 1549-1554.

11. Moshfegh AJ (2008) Research to advance understanding of the interrelationship of poverty and nutrition. $J$ Am Diet Assoc 107, 1882-1885.
12. Morland K, Wing S, Diez Roux A et al. (2002) Neighborhood characteristics associated with the location of food stores and food service places. Am J Prev Med 22, 23-29.

13. Moore L \& Diez Roux A (2006) Associations of neighborhood characteristics with the location and type of food stores. Am J Public Health 96, 325-331.

14. Lucan S, Karpyn A \& Sherman S (2010) Storing empty calories and chronic disease risk: snack-food products, nutritive content, and manufacturers in Philadelphia corner stores. J Urban Health 87, 394-409.

15. Bodor J, Rose D, Farley T et al. (2007) Neighbourhood fruit and vegetable availability and consumption: the role of small food stores in an urban environment. Public Health Nutr 11, 413-420.

16. Franco M, Diez-Roux A, Nettleton J et al. (2009) Availability of healthy foods and dietary patterns: the Multi-Ethnic Study of Atherosclerosis. Am J Clin Nutr 89, 897-904.

17. Morland K, Wing S \& Diez-Roux A (2002) The contextual effect of the local food environment on residents' diets: the atherosclerosis risk in communities study. Am J Public Health 92, 1761-1767.

18. Zenk SN, Lachance LL, Schulz AJ et al. (2009) Neighborhood retail food environment and fruit and vegetable intake in a multiethnic urban population. Am J Health Promot 23, 255-264.

19. Healthy Corner Store Network (2011) Healthy Corner Stores Tip Sheet. http://healthycornerstores.org/resources/ tip-sheets/ (accessed June 2011).

20. Borradaile K, Sherman S, Vander Veur S et al. (2009) Snacking in children: the role of urban corner stores. Pediatrics 124, 1293-1298.

21. Gittelsohn J, Song H, Suratkar S et al. (2010) An urban food store intervention positively affects food-related psychosocial variables and food behaviors. Health Educ Behav 37, 390-402.

22. Hartford Poverty Data (2011) Hartford, CT Poverty Rate Data - Information about poor and low-income residents. http://www.city-data.com/poverty/poverty-HartfordConnecticut.html (accessed February 2011).

23. McLaughlin T, Maljanian R \& McCormack K (2003) Hartford Health Survey 2003. Hartford, CT: The Institute for Outcomes Research and Evaluation, Hartford Hospital.

24. US Department of Agriculture, Economic Research Service (2010) Food Security Briefing Room. http://www.ers.usda. gov/Briefing/FoodSecurity/ (accessed November 2010).

25. SUPERVALU Inc. (2011) Annual Report Fiscal 2011. http:// www.supervalu.com/sv-webapp/downloads/SUPERVALU_ Fiscal_2011_Annual_Report.pdf (accessed September 2011).

26. Raudenbush SW, Spybrook J, Liu X et al. (2005) Optimal Design for Longitudinal and Multilevel Research, Version 1.55, Software Documentation. ftp://ftp.lisrel.com/hlm/ windows/downloads/OD_MANUAL.PDF (accessed November 2011).

27. Glanz K, Sallis J, Saelens B et al. (2007) Nutrition Environment Measures Surveys in stores (NEMS-S): development and evaluation. Am J Prev Med 32, 282-289.

28. Hartford City Data (2011) Hartford, Connecticut. http:// www.city-data.com/city/Hartford-Connecticut.html (accessed August 2011).

29. Song H-J, Gittelsohn J, Kim M et al. (2009) A corner store intervention in a low-income urban community is associated with increased availability and sales of some healthy foods. Public Health Nutr 12, 2060-2067. 